\title{
Vertex Models and Random Labyrinths: Phase Diagrams for Ice-type Vertex Models.
}

\author{
Kirill Shtengel 1 , ${ }^{4}$ and L. Chayes ${ }^{2}$ \\ ${ }^{1}$ Microsoft Research, One Microsoft Way, Redmond, WA 98052 \\ ${ }^{2}$ Department of Mathematics, University of California, Los Angeles, CA 90095-1555
}

(Dated: October 29, 2018)

\begin{abstract}
We propose a simple geometric recipe for constructing phase diagrams for a general class of vertex models obeying the ice rule. The disordered phase maps onto the intersecting loop model which is interesting in its own right and is related to several other statistical mechanical models. This mapping is also useful in understanding some ordered phases of these vertex models as they correspond to the polymer loop models with cross-links in their vulcanised phase.
\end{abstract}

\section{INTRODUCTION}

Ice-type models were originally introduced in order to describe the properties of ice [1, 2], and have been later generalised to represent other types of hydrogen-bonded crystals [3]. In ice, the oxygen atoms form a lattice with the coordination number of four. Each bond of the lattice contains a single hydrogen atom that is shifted from the middle point toward one of the neighbouring oxygen atoms. It has been conjectured, on the basis of local electro-neutrality, that precisely two hydrogen atoms are located near each oxygen atom with the other two being shifted away from it. This rule is known as the ice rule, and it can be graphically represented by placing polarisation arrows along the hydrogen bonds: For each site on the lattice there are exactly two incoming and two outgoing arrows. There are six such possible arrow configurations at each site which leads to another common name for this model (or rather its two-dimensional square lattice version): the sixvertex model. Interestingly, the states of ice-type models can be characterised by topological winding numbers and the excitations are topological defects carrying fractional charge [4], hence the recent revival of interest to this type of models in the context of topological order and fractionalisation in quantum systems [5, 6, 7]. The quantum phase transition in the quantum square lattice six-vertex model have been argued to mimic the physics of the HTSC materials with the $d$-density wave order while their counterparts on the triangular lattice twenty-vertex model could be relevant to the physics of 2D Wigner crystals [8].

The six-vertex model on the square lattice was solved exactly by Lieb [9, 10, 11, 12] and Sutherland [13] using the Bethe Ansatz and also (for some cases) by mapping it to a soluble free fermion problem [14]. For a review see [15, 16]. Much less is known about the ice-rule models in other contexts. For example, in the "next" 2D model, namely the twenty-vertex model on the triangular lattice, the Bethe Ansatz only works in a few instances when there are special relations between the parameters [17, 18, 19]. More importantly, there are very few broad-based techniques of general applicability with which these sorts of models can be studied.

In this paper we develop a new approach to establishing

shtengel@microsoft.com and understanding the phase diagrams of vertex models with the ice rule (i.e. equal numbers of incoming and outgoing arrows at each vertex). We introduce a new class of polymer models which is closely related to loop models, recently a topic of intensive study (see [20] and references therein). While a lot of progress has been achieved in studying loops models by the means of exact solutions [21] and conformal field theory [22], in the present analysis we will not recurse to those methods. The proposed general model is closely related to so-called Lorentz lattice gas [23, 24, 25, 26] or random labyrinths [27] as well as the ice-rule vertex models as will be discussed below. Such unified graphical representation on which our approach is based, is not entirely new, it has been discovered in the context of a loop algorithm developed to simulate vertex models[28, 29]. Notwithstanding the computational benefits inherent in this representation, here we will concentrate on the physical insight into the problem and the theoretical benefits which it provides. Indeed, our perspective allows for a very intuitive approach to a general class of vertex models and opens new possibilities for their rigorous analysis. This approach is ideologically similar to that of Fortuin and Kasteleyn [30] for the case of the Potts model.

Since a lot is known about the square lattice six-vertex model, this is a good reference point from which to start. We begin by reviewing a connection between the six-vertex and an intersecting loop model and show how the phase diagram can be immediately inferred. We then apply our method to a particularly interesting case of the twenty-vertex model identifying its phase boundaries. A more complete treatment of this problem with necessary proofs and additional examples shall be presented elsewhere [31].

\section{THE SWITCH MODEL}

Let us first introduce a statistical model of objects that we call switches. Consider a finite lattice $\mathbb{L}$ with an even coordination number. For simplicity let us discuss the case of a homogeneous lattice and, in particular, postpone the consideration of boundary sites. A switch is a variable associated with a lattice site. It is defined as a sorting of all incident edges into associated pairs. A more formal definition is as follows: Consider a complete graph $G(i)$ whose vertices are the bonds $\langle i, j\rangle$ of $\mathbb{L}$ connected to the lattice site $i$. A switch $\alpha_{k}(i)$ is a perfect matching in $G(i)$. Clearly, if $2 m$ is the coordination 
number, there are $(2 m-1)$ !! possible switches for every lattice site. On the square lattice, the three possible switches, the $\alpha$-switch, the $\beta$-switch and the $\gamma$-switch are shown in Fig. 1 In Ref. [27], in the context of random labyrinths, these have been referred to as NW and NE mirrors and tunnels respectively..
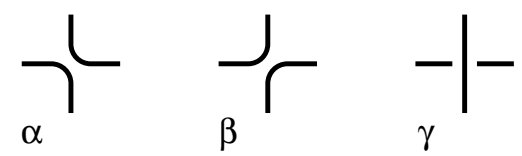

FIG. 1: The three possible switches for the two-dimensional square lattice. We do not distinguish between under- and over-crossings for the gamma-switches (we are not concerned with topology for the purposes of this paper).

In general, we shall call the switches the $\alpha_{1}$-switch, the $\alpha_{2}$-switch etc. We will denote by $\mathcal{S}$ a configuration of such switches on $\mathbb{L}$. The weight of $\mathcal{S}$, which we denote by $Q(\mathcal{S})$ is given as follows: First, there are a priori weights (or activities) associated with every type of switches. These will be conveniently denoted by $\alpha_{1}, \alpha_{2}$ etc. Next, we observe that $\mathcal{S}$ divides the bonds of the lattice into loops. Indeed, each bond $\langle i, j\rangle$ is a member of two switches $\alpha_{k}(i)$ and $\alpha_{k^{\prime}}(j) \in \mathcal{S}$ which pair it with two other bonds etc. We can continue growing this path in both directions until the loop is closed, i.e. the two "end" bonds are paired by a switch. A loop is thus a cycle of bonds such that every two adjacent bonds are paired up by a corresponding switch in $\mathcal{S}$. We are not distinguishing the cycles different only by a reversal of the overall order. Notice that in general such loop can visit the same site more than once and in particular, due to tunnels, can have multiple self-intersections. We let $\ell(S)$ denote the number of such loops and $\mathrm{A}_{1}(S), \mathrm{A}_{2}(S), \ldots$ the number of $\alpha_{1}$-switches, $\alpha_{2}$ switches etc. Then $Q$ is given by

$$
Q=\prod_{k=1}^{p} \alpha_{k}^{\mathrm{A}_{k}(\mathcal{S})} n^{\ell(\mathcal{S})}
$$

where $p=(2 m-1) !$ ! is the total number of distinct switches and $n$ is a positive real number. If $n$ is an integer, it can be thought of as a number of different colours available for every loop. The partition function is simply

$$
Z \equiv Z_{\alpha_{1}, \ldots, \alpha_{S}}=Z=\sum_{S} Q(S) .
$$

We also introduce a simple extension of the above switch model in which we allow two (or more - depending on the lattice) paths at a given site to be "fused" together. We shall refer to such objects as cross-links. In general, the cross-links may be partial in the sense that not all pairs of bonds adjacent to a site are fused. For a four-coordinated lattice such as the 2D square lattice, there naturally can be only a single type tying up all four adjacent bonds. Aside from associating the additional a priori weight $\phi$ with such cross-links, their introduction results in the following. If $n$ is an integer, loops that have been fused together are now required to have the same colour. For an arbitrary $n$ this translates into the requirement that all such fused loops count as one for the purposes of evaluating the corresponding weights in Eq. (1).

As has already been mentioned, the switch model and its extension are closely related to several known statisticalmechanical models.

The $n \rightarrow 0$ limit of the switch model describes the Eulerian walks or cycles (depending on the boundary conditions), i.e. walks that traverse every bond exactly once. Self-intersections are allowed via tunnels. If one were to adopt such a model for describing a polymer, the weight for the tunnels should translate into the rigidity of a polymer molecule.

The $n=1$ case corresponds to Lorentz lattice gas [23, 24, 25, 26] or random labyrinths [27]. The model in [27] on a hypercubic lattice $\mathbb{Z}^{d}$ is nearly identical to our extended model, with the following difference: instead of cross-links, there are normal sites at which a path that is otherwise consistent with the switches can randomly, with equal probability, go in any of the $2 d$ directions. This is equivalent to constructing a random walk on a graph whose vertices are the cross-links and edges are the switch-mediated paths between them. The model corresponds to $n=1$ since the switches (mirrors and tunnels) as well as cross-links (normal sites) are distributed with independent probabilities.

For arbitrary $n$, if all switch weights are identical, this is just a fully-packed limit of the $\mathrm{O}(n)$ loop model [32].

A special integrable case of a model (11) with $n<2$ corresponds to a supersymmetric spin chain [33]

Finally, the $n=2$ case corresponds to the ice-rule vertex models, this is the subject of the following section.

\section{RELATION TO THE ICE-TYPE MODELS}

A mapping between $n=2$ switch models and and ice-type models is easiest seen in the case of the square lattice sixvertex model whose possible vertices are shown in Figure 2 Each of those vertices is assigned a corresponding energy $\varepsilon_{i}$

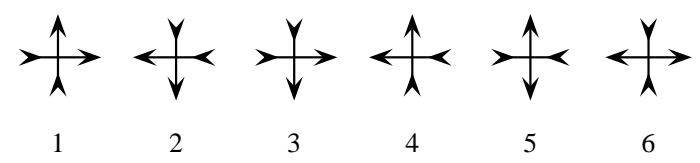

FIG. 2: The six vertices allowed by the ice rule.

$(i=1, \ldots, 6)$. In the absence of external electric field, the model should be invariant under the reversal of all arrows thus requiring $\varepsilon_{1}=\varepsilon_{2}, \varepsilon_{3}=\varepsilon_{4}$ and $\varepsilon_{5}=\varepsilon_{6}$. For the remainder of this paper we shall concern ourselves not with the energies as such, but rather with the (unnormalised) Boltzmann weights $w_{i} \propto \exp \left(-\beta \varepsilon_{i}\right)$. Conforming to to the usual notations (cf. [15, 16]), we shall use $a=w_{1}=w_{2}, b=w_{3}=w_{4}$ and $c=w_{5}=w_{6}$.

The mapping of the switch model to the six-vertex model is done by assigning directions to all loops (since there are two choices for every loop, the factor of $2^{\ell}$ in Eq. (1) can be obtained by summing over all such choices). Tracing out the switch variables as illustrated in Fig. 3 a) leaves us with the 
six-vertex configurations [47]. The weights are given by

$$
a=\beta+\gamma, \quad b=\alpha+\gamma, \quad c=\alpha+\beta
$$
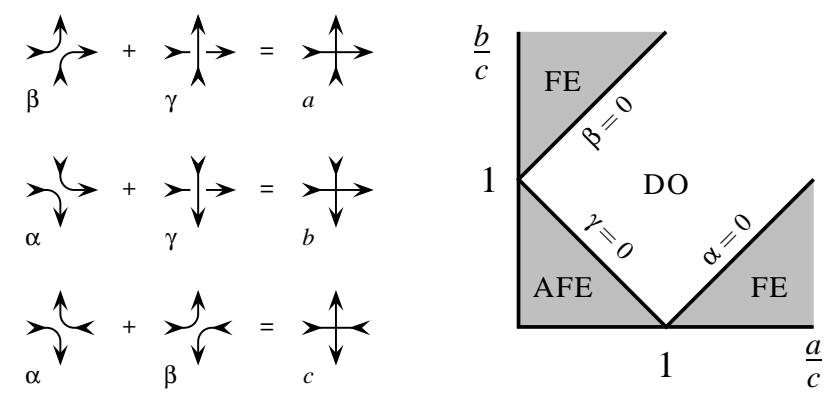

FIG. 3: (a) Tracing out switches by fusing directed loop segments into the ice-type vertices (only types 1,3 and 5 are shown, types 2, 4 and 6 are obtained by merely reversing all arrows). (b) The phase diagram of the six-vertex model.

In general, we claim that the partition function for the switch model with weights (1) where $n=2$ is exactly the partition function for an ice-rule vertex model with vertex weights determined by

$$
w_{i}=\sum_{k} \alpha_{k} \Delta_{i}^{k}
$$

where $\Delta_{i}^{k}=1$ if the vertex $V_{i}$ and the switch $S_{k}$ are consistent and is 0 otherwise. $V_{i}$ and $S_{k}$ are consistent if there is one incoming and one outgoing arrow for each pairing of the switch. (Notice that this automatically preserves the ice rule.) The most direct way to see this is to consider a joint measure on both a switch configuration $\mathcal{S}$ and an arrow configuration $\mathcal{A}$ with the weight

$$
\tilde{Q}=\prod_{k=1}^{p} \alpha_{k}^{\mathrm{A}_{k}(\mathcal{S})} \Delta(\mathcal{S}, \mathcal{A})
$$

where $\Delta(\mathcal{S}, \mathcal{A})=1$ if $\mathcal{S}$ and $\mathcal{A}$ are consistent in the above sense and 0 otherwise. Now, summing this weight over all possible arrow configurations $\mathcal{A}$ leads to the the weight of $\mathcal{S}$ given by Eq. (1) while tracing out switches leaves us with ice-type arrow configurations where the weight for a single vertex is given by Eq. (4).

We now take a closer look at Eqs. (3) and notice that if $\alpha, \beta, \gamma>0$ (and the switch model only makes sense for nonnegative weights), the allowed values of $a, b$ and $c$ lie precisely within the entire disordered (DO) region of the sixvertex model (Fig. 3 b)). In fact, we argue, this is not a coincidence but a general case: a switch model with all switch weights positive always maps onto a DO phase of a vertex model [48]. But before we present our argument, we should discuss the boundary conditions which are defined as follows: let us regard our lattice $\mathbb{L}$ as actually sitting inside the larger (possibly infinite) $\Lambda$. Let $\partial \mathbb{L}$ denote those bonds that connect a site in $\mathbb{L}$ with a site outside. A boundary condition is defined as a constraint on these bonds' colours (for $n$ integer) or their external connectedness (general $n$ ). From the perspective of the partition function, we note that the paths in $\mathbb{L}$ either form closed loops or end at the boundary; each boundary bond now serves as a "source/sink" for a path.

There are several rules that can be now invoked: The simplest two - which are the extreme cases - are (i) all such paths count as additional loops in Eq. (1) for the weight of a given switch configuration, or (ii) the only loops counted are the internal loops. There are also many intermediate sets of rules as well as superpositions of various boundary conditions, each with their own set of rules. Both free and periodic boundary conditions are examples of such superpositions. It is noted that none of the above amounts to any internal constraints they are all "external constraints", i.e. all internal switch configurations $\mathcal{S}$ are legitimate. This is in sharp contrast to boundary conditions that are imposed by placement of arrows. Indeed, consider the six-vertex model on a cylinder of girth $L$. If $m$ upward arrows and $L-m$ downward arrows are placed on the bottom boundary, then the only allowed switch configurations are those in which there are $|L-2 m|$ paths that start at the bottom and end at the top. Under such circumstances, it is easy to see that boundary conditions are capable of affecting the bulk free energy.

What about the order parameter for the switch model? For an integral $n$, the natural choice is the expectation value for a bond at the origin to be of a particular colour. Similarly to the Fortuin-Kasteleyn (FK) random cluster model, this translates into the probability that a given bond at the origin is connected to the boundary; with this definition $n$ needs not remain an integer. Two bonds are considered connected if and only if there exist a switch-mediated path of bonds between them (using cross-links is also allowed in constructing such path). In the absence of cross-links, this means that the two bonds belong to the same loop. If cross-links are allowed, the two bonds must belong to the same cluster of fused together loops.

However, there is an important difference between the order parameter in the FK model and that in our model. While in the integer $q \mathrm{FK}$ model, the probability that the origin is connected to the boundary is (at least for the wired boundary conditions) equal to the spontaneous magnetisation of the corresponding Potts model [34], in our model such relation between the graphical and the physical order parameters becomes more complicated. To illustrate this point, consider again the six-vertex model. Unlike the Ising magnet, it actually has two ordered phases, ferroelectric (FE) and antiferroelectric (AFE) that are not related to each other by a simple gauge transformation. While for each given instance of the six-vertex model only one of these ordered phases can be accessed by the means of lowering temperature (which we have absorbed in the vertex weights), there are two possible order parameters with no obvious relation between them. One of them, namely the uniform polarisation is discussed in Appendix $\mathrm{A}$ For now it suffices to note that connectedness to the boundary is a necessary, if not sufficient condition for any type of physical order; the internal loops can be reversed at will and thus do not contribute to a long-range order. Along the same lines, we note that any two locally defined quantities at two locations can be correlated only if the corresponding 
bonds belong to the same loop. The nature of such correlation is trivial in terms of colours but may be much more complicated in terms of arrows. In this sense, the probability of two bonds to belong to the same loop is an upper bound for any (truncated) correlation function.

Let us now present a physical (albeit non-rigorous) argument supporting our earlier statement that a $n=2$ switch model with all switch weights positive is always in a DO phase. All we have to show is that less than a finite fraction of bonds in the interior are connected to the boundary. We start by considering a $n=1$ model in an upper half-space. Because $n=1$, we do not need to count loops and may construct the partition function by "growing" our system upward from the boundary layer-by-layer, the choice for each switch being completely independent of others. In such a setting it is easy to see that the assumption of a finite limit of density of (vertical) bonds connected to the bottom is inconsistent with the finite probability of terminating any two such strands with finite separation between them (by turning paths toward each other with the analogs of $\alpha$ - and $\beta$-switches and then connecting them via a finite chain of tunnels) at every given level. This argument does not preclude a power-law or even logarithmic decay of such density away from the boundary, and in fact this is precisely what has been observed numerically [24]. What about $n=2$, the case of our primary interest? Lacking a proof (the previous part of the argument can be made rigorous [31]), one can nevertheless argue that the $n>1$ case always favours shorter loops thus strengthening the above conclusion [49]. Notice that there is nothing particularly 2D about this argument, we expect our criterion to work in any dimensionality.

Let us now turn to the ordered phases. As follows from the above discussion, a long-range order can not appear unless one of the switch weights vanishes. To see that this condition indeed correctly describes the boundary, it is instructive to look at the six-vertex model again. Let us start with the FE phase boundary, e.g. the one described by $b=a+c$ or, in our language, $\beta=0$. Notice, that a line going up can now either continue going up passing through $\gamma$-switches or turn to the left via an $\alpha$-switch, upon which it will continue to the left until the next $\alpha$-switch will send it up again. It can never turn back on itself! Thus, the total number of loops $\ell$ in Eq. (1) in an $L \times L$ sample is at most $2 L$ and scales sublinearly with the size of the system $N=L^{2}$. As a result, in the thermodynamic limit, the factor of $2^{\ell}$ in Eq. (1) is inconsequential and can be dropped. Then the partition function is trivially calculated as

$$
Z=\sum_{S \in\{\alpha, \gamma\}^{N}} \alpha^{A} \gamma^{N-A}=(\alpha+\gamma)^{N}=b^{N}
$$

meaning that the system becomes fully frozen: ether all vertices are of type 3 or of type 4 . Notice that once again, this argument is not specifically 2D and could be easily used in higher dimensions, e.g. it generalises the proof by Nagle [35] of a first order transition in a KDP model on d-dimensional tetrahedral lattices. Inside this phase, i.e. when $b>a+c$, we need to extend the model by allowing cross-links so that $b=\alpha+\gamma+\phi, a=\gamma, c=\alpha$ (this is in fact very similar to "freezing" a vertex in the loop algorithm of [28]). Notice that in general a cross-linked model is not automatically equivalent to an ice-type vertex model. One has to verify that a consistent arrow assignment can be done for all cross-linked clusters with the cross-links corresponding to the excessive vertices. For the FE phase, cross-linking the switch model is automatic, but not particularly enlightening since the model fully orders already at the transition point. The AFE phase presents a more interesting case. At the boundary of the AFE phase $\gamma=0$, i.e. loops turn at every step and cannot selfintersect. Assigning directions renders each such loop consistent with a perfect staggered AFE order, but since each loop is free to choose one of the two possible directions, no overall order results. Moving into the phase, we once again resort to cross-links to compensate for excessive $c: c=\alpha+\beta+\phi$, $a=\beta, b=\alpha$. Cross-links do not change the geometry of loops but rather "vulcanise" them into clusters. It is easy to verify that such clusters are always consistent with one of the two possible staggered polarisations. What is essential here is the fact that in the DO phase the loops are critical, as expected in this type of models on general grounds. Therefore such vulcanisation leads to percolation of a particular colour or, alternatively, staggered polarisation throughout the sample. What is a bit puzzling, is that the AFE transition is actually of the infinite order, not of the second order as our naive argument would generally suggest; this must be due to the criticality of the phase on the DO side but we do not have a clearer picture for that in terms of loops.

\section{TWENTY-VERTEX MODEL ON THE TRIANGULAR LATTICE}

So far we dealt with the lattice of coordination number four. This is somewhat special since the number of possible vertices (up to the reversal of all arrows) allowed by the ice rule is the same as the number of possible switches - three. This is not generally the case. Let us turn our attention to the triangular lattice. Its coordination number is six which means there are $\left(\begin{array}{l}6 \\ 3\end{array}\right)=20$ distinct ways of arranging tree inward and tree outward pointing arrows. By requiring that the weights for the vertices are arrow-reversal invariant (zero electric field condition), the number of parameters is reduced to 10 . On the other hand, there are $5 ! !=15$ distinct switches. Therefore Eqs. (4) form an underdetermined system. So, at a first glance the situation appears hopeless: we cannot even uniquely solve this system, so how could we expect to deduce the phase boundaries? Nevertheless, the proposed criterion stands: as long as it is possible to satisfy Eqs. (4) with positive switch weights, the system is in its (critical) DO phase. To illus-

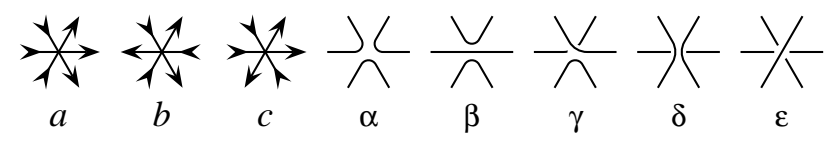

FIG. 4: The distinct (up to rotations and reflections) vertices and switches on the triangular lattice with their weights. The third vertex is chiral, with only one chirality shown; both have the same weight. 
trate this, consider the symmetric version of the twenty-vertex model (dubbed the F-model in [17]) by requiring that all vertex weights are invariant under the symmetry group $\mathrm{D}_{6}$. This leaves us with only three distinct types of vertices and five types of switches depicted in Fig. 4 Eqs. (4) read:

$$
\begin{array}{r}
a=\beta+2 \gamma+2 \delta+\varepsilon, \\
b=2 \alpha+3 \beta+\varepsilon, \\
c=\alpha+\beta+3 \gamma+\delta,
\end{array}
$$

or, equivalently

$$
\begin{array}{r}
a+2 c=b+8 \gamma+4 \delta, \\
b+2 c=a+4(\alpha+\beta+\gamma), \\
b+3 a=2 c+4(\beta+\delta+\varepsilon) .
\end{array}
$$

From the latter form, it is clear that there are no solutions with all switch weights positive unless $a+2 c>b, b+2 c>a, b+$ $3 a>2 c$; according to our criterion this is the DO region of the model. The suspected phase boundaries correspond to $\alpha=$ $\beta=\gamma=0, \gamma=\delta=0$ and $\beta=\delta=\varepsilon=0$. These conditions have a simple geometric interpretation in terms of loops: in the first case, connections of bonds at $60^{\circ}$ are excluded, in the second, the are no connections at $120^{\circ}$ and in the third, there are no direct tunnels. We now turn to the suspected ordered

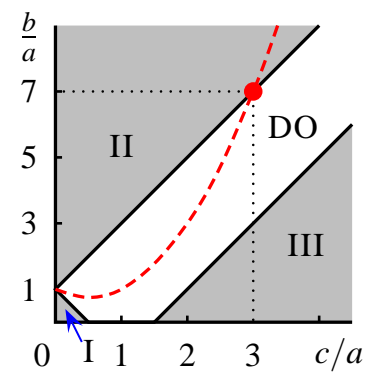

(b)

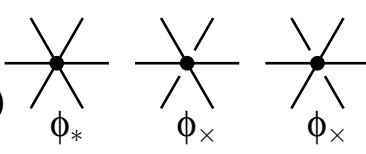

(c)

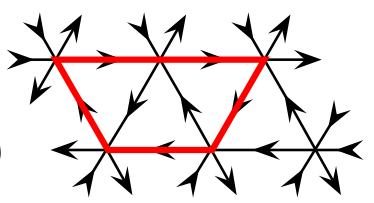

FIG. 5: (a) The proposed phase diagram for the F-model on the triangular lattice shown. Along the dashed line the Bethe Ansatz solution is available with the known transition point circled. (b) Complete and partial cross-links important for understanding the ordered phases II and I (see text for details). (c) A zero mode in a state composed entirely of $c$-vertices; the arrows along the selected pentagon can be freely reversed.

phases. The simplest one is the phase II $(b>a+2 c)$; it is easy to verify that the introduction of complete cross-links $\left(\phi_{*}\right.$ in Fig. 5b) corresponding to excessive $b$-vertices is consistent with the onset of the spontaneous sextipole electric moment - a triangular flux phase. This is the only ordered phase of the model accessed by the means of exact solutions [15, 17] available along the dashed line in Fig. $5 \mathrm{~b}$, a transition to the ordered phase was found to be of the infinite order.

In the phase I $(a>b+2 c)$, on the other hand, complete cross-links are inconsistent with the dipolar order described by the proliferation of $a$-vertices; such cross-link corresponding to any particular $a$-vertex cannot be broken into all three different $\delta$-switches. Physically, this inconsistency arises from the fact that the symmetry of the ordered phase (mod complete arrow reversal) is lower than the symmetry of the cross-link and switch weights. One possible remedy is to replace complete cross-links with the partial ones $\left(\phi_{\times}\right.$in Fig. 5b) breaking such symmetry by hand. The particular choice of two out of three possible partial switches prefers a horizontal dipolar order: $a_{-}=2 \delta+\varepsilon+2 \phi_{\times}, a_{\searrow}=a_{/}=2 \delta+\varepsilon+\phi_{\times}$ where the subscripts for $a$-vertices indicate directions of their middle out-arrows. Now once again, vulcanisation of loops leads to the onset of the dipolar order in the vertex model, but this is not the original F-model, we have reduced the symmetry of the $a$-vertex weights. We nevertheless expect a similar transition to occur in the F-model since the two models are identical at the transition line of the modified model and their ordered phases have the same broken symmetry [50]. A plausible counter-argument would point at the criticality of the DO phase making the above reasoning suspect: moving in the "wrong" direction on the phase diagram can easily order a critical phase while the system might remain disordered if we carefully tread in the "right" direction. We argue, this is not the case: the disordered phase here actually expected to have finite susceptibility to the uniform electric field as is the case for the six vertex model [15, 36] [51]. Therefore the non-analytic onset of the dipolar order in any direction of the phase diagram signifies a true phase transition.

Finally, there is Region III $(2 c>b+3 a)$ described by the abundance of $c$-vertices. Cross-linking the loops does not appear to work here since unlike in the previous two cases, some ground states of system have local zero modes (Fig.57). What is even more unsettling, is that in terms of loops a particular zero mode depicted in Fig. 55 contains a direct tunnel, something that disappears on the boundary of Region III but seemingly reappears well inside. We therefore can only speculate on the nature (and the existence) of the transition into this phase, although the fact that a certain class of loops that could freely fluctuate in the DO phase disappears at the boundary remains very suggestive. One possibility is the onset of an entropically stabilised phase, i.e. an order-by-disorder transition; further investigation is needed to clarify this issue.

\section{TWENTY-VERTEX MODEL ON THE CUBIC LATTICE}

Interestingly, there is a one-to-one correspondence between the vertices (and corresponding switches) in twenty-vertex models on the 2D triangular and 3D cubic lattice (it becomes obvious if one identifies $\pm 60^{\circ}$ directions in Fig. 4 with $y$ - and $z$-axes). Reducing the cubic lattice model to its F-model version by requiring that all vertex weights are invariant under $\mathrm{O}_{h}$ (the point symmetry group of the cubic lattice), we are left with only two vertex weights $a$ and $c$ (in notations of Fig. 4 $b \equiv a)$ and three switch weights $\alpha, \beta$ and $\varepsilon(\gamma \equiv \alpha$ and $\delta \equiv \beta)$. It then follows from Eqs. (4) that $2 a=c+4 \beta+2 \varepsilon$. If $2 a>c$, the model is in the DO phase. At $2 a=c$ we have $\beta=\varepsilon=0$ meaning that loops must turn at every step, this is a $3 \mathrm{D}$ analog of non-self-intersecting loops. Every loop is perfectly AFEordered, and as we have seen, on a 2D square lattice this signifies a transition to the AFE phase. There is also a general statement about self-intersections being a relevant perturba- 
tion in a similar type of 2D loop models [37] suggesting that the non-intersecting fully-packed loops characterise an unstable fixed point. So once again, it is suggestive that $2 a=c$ is a transition point. In 3D, however, the region with $2 a<c$ is analogous to Region III of a triangular lattice model. In particular, there are zero modes in some of its ground states similar to that shown in in Fig. 55 (naturally, these modes corresponds to the loops of even length now). Thus, sorting out the properties of Region III of the F-model on the triangular lattice might shed some light on the properties of the cubic F-model.

\section{CONCLUSION}

To conclude, we introduced a class of intersecting polymer loop models with the loop fugacity $n$. For $n=2$, such a model is equivalent to an ice-type vertex model in its DO critical phase. We conjecture that the boundaries of the DO phase are encountered at the parameter values which force one or more switch weights to vanish. The onset of a long-range order in a vertex model is often, but not always equivalent to the formation of cross-links between polymer loops that results in a vulcanisation transition. This correspondence is not strictly 2D, although in this paper we concentrated mostly on 2D examples to benefit from the additional information available from the exact solutions. We note that all known transition points in the ice-rule models satisfy our criterion. As an additional benefit, we can understand transitions on irregular lattices [52].

However, despite its suggestive nature, it remains a big challenge to turn our criterion into a rigorous statement.

\section{Acknowledgments}

It is our pleasure to acknowledge helpful discussions with T. Prellberg, J. F. Nagle, M. den Nijs, P. Fendley and C. Nayak.

\section{APPENDIX A: UNIFORM POLARISATION AND SUSCEPTIBILITY.}

Consider an $L \times L$ cylinder (or its generalised $d-$ dimensional version - an $L \times L \times \ldots \times L$ lattice with periodic boundary conditions imposed in $d-1$ dimensions). The underlying lattice needs not be square or even regular as long as appropriate periodic boundary conditions can be imposed. The boundary condition in the remaining direction, i.e. along the cylinder axis is left free. We now apply a uniform electric field $\mathbf{E}$ along the axis. The coupling to this field is assumed to be of the standard form: $\Delta H=-\sum_{\langle i j\rangle} \mathbf{E} \cdot \mathbf{d}_{\mathbf{i j}}$ where $\mathbf{d}_{\mathbf{i j}}$ is a vector in the direction of the arrow along the $\langle i j\rangle$ bond with the magnitude proportional to the length of the bond (for a regular lattice it is sufficient to choose all $\left|\mathbf{d}_{\mathbf{i j}}\right|=1$ ). Because of the last condition, $\sum_{\langle i j\rangle \in \bigcirc} \mathbf{E} \cdot \mathbf{d}_{\mathbf{i j}}=0$ along any closed loop on the lattice. This allows one to evaluate the partition function of the vertex model in the form similar to Eqs. (11,2):

$$
Z=\sum_{S} \prod_{k=1}^{p} \alpha_{k}^{\mathrm{A}_{k}(\mathcal{S})} 2^{\ell(\mathcal{S})}(2 \cosh \beta E L)^{m(\mathcal{S})}
$$

where $\ell(\mathcal{S})$ is the number of closed loops while $m(\mathcal{S})$ is the number of paths connecting the top and the bottom boundaries - see Fig. 6 The switch weights in Eq. A1 correspond to the vertex weights in the absence of an external field.

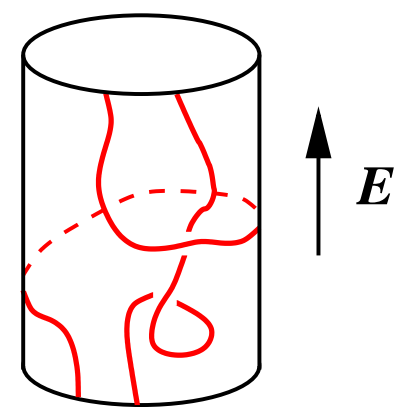

FIG. 6: Only paths connecting the top and the bottom of the cylinder can be polarised by the electric field parallel to its axis. The contribution of each such paths is proportional to the cylinder height regardless of the path's geometry.

The uniform polarisation per site is then

$$
p=\frac{1}{N \beta} \frac{\partial \ln Z}{\partial E} \propto L^{1-d}\langle m\rangle \tanh \beta E L \underset{E \rightarrow 0}{\longrightarrow} L^{2-d} \beta E\langle m\rangle
$$

for small field. Here $\langle m\rangle$ is the expected number of top-tobottom paths. The uniform susceptibility is then

$$
\chi=\left.\frac{\partial p}{\partial E}\right|_{E=0} \propto L^{2-d}\langle m\rangle .
$$

An extensive study of the square lattice six-vertex model in a uniform electric field presented in Ref. [15] suggests that this model has a finite non-zero susceptibility to such field everywhere in the DO region. According to Eq. A3 this implies that $\langle m\rangle$ remains finite in the thermodynamic limit. The fact that a probability of a path connecting the opposite sides of an $L \times L$ sample remains finite as its size grows is consistent with the $r^{-2}$ decay of the loop correlation function. This is indeed the case for the points soluble via a free fermion mapping [36, 38]. On the other hand, a mapping of the six-vertex model to an effective Gaussian model (via a restricted solidon-solid (RSOS) model) and a Coulomb gas [39] leads one to think that the exponent characterising the power law decay of correlations continuously changes throughout the DO phase. We notice, however, that the intersecting loops defined by the switch model are not the same as the non-intersecting loops running along the steps of equal height in the RSOS model which are described by the "magnetic" exponents in the Coulomb gas language. It must be the case then that the proper correlation function for our loops is described by a combination of magnetic and electric operators "conspiring" to keep its $r^{-2}$ long-distance behavior throughout the DO region. 
We finally remark that it has been conjectured in Ref. [40] that an arrow-arrow correlation function for a generic ice-rule model decays as $r^{-d}$ (although, it seems, the basis for this claim is an exact result of [38] whose scope is limited to the free-fermion line of the 2D six-vertex model). This conjecture appears natural in light of Eq. A3): such behaviour is necessary for $\langle m\rangle \sim L^{d-2}$ which in turn is required for a finite non-zero susceptibility to a uniform electric field.
[1] J. D. Bernal and R. H. Fowler, J. Chem. Phys. 1, 515 (1933).

[2] L. Pauling, J. Am. Chem. Soc. 57, 2680 (1935).

[3] J. C. Slater, J. Chem. Phys. 9, 16 (1941).

[4] M.-S. Chen, L. Onsager, J. Bonner, and J. Nagle, J. Chem. Phys. 60, 405 (1974).

[5] M. Hermele, M. P. A. Fisher, and L. Balents (2003), condmat/0305401.

[6] E. Ardonne, P. Fendley, and E. Fradkin, cond-mat/0311466, to appear in Ann. Phys.

[7] E. Fradkin, D. A. Huse, R. Moessner, V. Oganesyan, and S. L. Sondhi (2003), cond-mat/0311353.

[8] S. Chakravarty, Phys. Rev. B 66, 224505 (2002).

[9] E. H. Lieb, Phys. Rev. 162, 162 (1967).

[10] E. H. Lieb, Phys. Rev. Lett. 18, 692 (1967).

[11] E. H. Lieb, Phys. Rev. Lett. 18, 1046 (1967).

[12] E. H. Lieb, Phys. Rev. Lett. 19, 108 (1967).

[13] B. Sutherland, Phys. Rev. Lett. 19, 103 (1967).

[14] C. Fan and F. Y. Wu, Phys. Rev. B 2, 723 (1970).

[15] E. H. Lieb and F. Y. Wu, in Phase Transitions and Critical Phenomena, edited by C. Domb and M. S. Green (Academic Press, London, 1972), vol. 1, pp. 331-490.

[16] R. J. Baxter, Exactly Solved Models in Statistical Mechanics (Academic Press, New York, 1982).

[17] R. J. Baxter, J. Math. Phys. 10, 1211 (1969).

[18] S. B. Kelland, J. Phys. A: Math. Gen. 7, 1907 (1974).

[19] S. B. Kelland, Aust. J. Phys. 27, 813 (1974).

[20] C. VanDerZande, Lattice Models of Polymers (Cambridge University Press, Cambridge, 1998).

[21] M. T. Batchelor, B. Nienhuis, and S. O. Warnaar, Phys. Rev. Lett. 62, 2425 (1989).

[22] J. Kondev, Int. J. Mod. Phys. B 11, 153 (1997), condmat/9607181.

[23] T. W. Ruijgrok and E. G. D. Cohen, Phys. Lett. A 133, 415 (1988).

[24] R. M. Ziff, X. P. Kong, and E. G. D. Cohen, Phys. Rev. A 44, 2410 (1991).

[25] L. A. Bunimovich and S. E. Troubetzkoy, J. Stat. Phys. 67, 289 (1992).

[26] M.-S. Cao and E. Cohen, J. Stat. Phys. 87, 147 (1997).

[27] G. R. Grimmett, M. V. Menshikov, and S. E. Volkov, Markov Processes and Related Fields 2, 69 (1996).

[28] H. G. Evertz, G. Lana, and M. Marcu, Phys. Rev. Lett. 70, 875 (1993).

[29] H. G. Evertz, Adv. Phys. 52, 1 (2003), cond-mat/9707221.

[30] C. M. Fortuin and P. W. Kasteleyn, Physica 57, 536 (1972).

[31] L. Chayes and K. Shtengel, in preparation.

[32] L. Chayes, L. P. Pryadko, and K. Shtengel, Nuclear Physics B 570, 590 (2000), cond-mat/9910292.

[33] M. J. Martins, B. Nienhuis, and R. Rietman, Phys. Rev. Lett. 81, 504 (1998), cond-mat/9709051.

[34] M. Aizenman, J. T. Chayes, L. Chayes, and C. M. Newman, J. Stat. Phys. 50, 1 (1988).

[35] J. F. Nagle, Commun. Math. Phys. 13, 62 (1969).

[36] R. J. Baxter, Phys. Rev. B 1, 2199 (1970).

[37] J. L. Jacobsen, N. Read, and H. Saleur, Phys. Rev. Lett. 90,
090601 (2003), cond-mat/0205033.

[38] B. Sutherland, Phys. Lett. A 26, 532 (1968).

[39] B. Nienhuis, in Phase Transitions and Critical Phenomena, edited by C. Domb and J. L. Lebowitz (Academic Press, London, 1987), vol. 11, pp. 1-53.

[40] A. Yanagawa and J. F. Nagle, Chem. Phys. 43, 329 (1979).

[41] A. Rahman and F. H. Stillinger, J. Chem. Phys. 57, 4009 (1972).

[42] B. Nienhuis, Int. J. Mod. Phys. B 4, 929 (1990).

[43] M. Aizenman and B. Nachtergaele, Commun. Math. Phys. 164, 17 (1994).

[44] G. T. Barkema and M. E. J. Newman, Phys. Rev. E 57, 1155 (1998), cond-mat/9706190.

[45] K. Rommelse and M. den Nijs, Phys. Rev. Lett. 59, 2578 (1987).

[46] M. den Nijs and K. Rommelse, Phys. Rev. B 40, 4709 (1989).

[47] An analogous construction for the case of square ice $(a=b=c)$ was described in our earlier paper [32]. In fact, similar ideas were previously discussed in Refs. [28, 29, 40, 41, 42, 43, 44], but with the exception of [42, 43]), they have not been used as a basis for analytical approach.

[48] This in never a truly disordered phase though; due to conserved "arrow currents", the correlations are always expected to be critical (even in higher dimensions) as is the case for the sixvertex model.

[49] We should mention here that at least in 2D, such loop model might still order if $n$ becomes sufficiently large: in our earlier paper [32] we actually proved this for a wide class of models that include the switch model on the square lattice with $\alpha=\beta$. This ordering, however, happens not because the loops become long but rather because they become extremely short. When the entropy associated with large $n$ forces most of the loops to become of length 4 , they break the translational symmetry of the lattice by circling plaquettes in a checkerboard pattern. Naturally, such transition is not associated with any colour symmetry breaking.

[50] The fully ordered phase of the F-model can have at most six non-collinear domain walls, meaning there will be macroscopic regions with dipolar order. Alternatively, it can have an unlimited number of collinear degrees of freedom: just think of a state in which all vertices correspond to the same choice of one of the two partial cross-links in Fig. 55. After the cross-linked cluster is assigned directions (so that outgoing arrows are at $60^{\circ}$ to each other), we can chose directions for the unlinked lines along the third axis independently at random. Notice, however, that there are at most $L$ such lines (for free boundary conditions) and therefore the ground state has zero entropy per site in the thermodynamic limit. It also has a long-range order with broken dipolar symmetry due to the cross-linked cluster.

[51] This is due to the fact that closed loops, albeit critically long, do not couple to the uniform field - see Appendix A The only contribution to the susceptibility must come from finitely many paths connecting the opposite boundaries of the sample. Notice that the existence of a finite number of such paths does not contradict our earlier argument for the DO phase which precluded a finite density of such paths. 
[52] E.g., the verticality of the line separating rough from flat phases as a function of step repulsion on the phase diagram for the RSOS model shown in [45, 46] becomes clear from our point of view: this is just a six-vertex model on a loop-diluted square lattice. The transition is determined by a local condition on the vertex weights and is independent of the degree of dilution for as long as the underlying lattice holds together. 